\title{
Diagonally implicit block backward differentiation formulas for solving fuzzy differential equations
}

\begin{abstract}
In this work, the diagonally implicit 2-points block backward differentiation formulas (DIBBDF) is developed for solving Fuzzy Differential Equations (FDEs) under the interpretation of generalized Hukuhara differentiability. The fuzzy configuration of this method is also introduced. Numerical results using DIBBDF are presented and compared with the existing method. It is clearly shown that the proposed method obtains good numerical results and suitable for solving FDEs.
\end{abstract}

Keyword: Block method; Diagonally implicit; Fuzzy; Hukuhara differentiability 